\title{
ÜBER RINGE MIT MINIMALBEDINGUNG FÜR HAUPTRECHTSIDEALE. II
}

\author{
Von \\ F. SZÁSZ (Budapest) \\ (Vorgelegt von L. RÉDEI)
}

Professor L. RédeI zu seinem 60. Geburtstag gewidmet

\section{$\S 1$. Einleitung ${ }^{1}$}

Die im Titel erwähnten Ringe werden kurz $M H R$-Ringe genannt, die schon in unserer Arbeit [26] untersucht worden sind. Unter einem Ring verstehen wir immer einen assoziativen Ring, unter dem Radikal das Jacobsonsche Radikal, unter einem halbeinfachen Ring einen Ring mit dem Radikal (0). Für die Grundbegriffe verweisen wir auf [13], ferner auf [1], [4], [7] und [21]. Unter einem $M H_{1} R$-Ring verstehen wir einen Ring mit Minimalbedingung für die in Hauptrechtsidealen liegenden Rechtsideale. Hiernach ist jeder Artinsche Ring (d. h. ein Ring mit Minimalbedingung für Rechtsideale) ein $M H_{1} R$-Ring und jeder $M H_{1} R$-Ring ist ein $M H R$-Ring, aber die Umkehrungen sind falsch. Ferner wird ein Ring mit Minimalbedingung für Hauptideale (bzw. für die in Hauptidealen liegenden Ideale) kurz MHI-Ring (bzw. $M H_{1} I$-Ring) genannt. Die $M H R$-Ringe mit Maximalbedingung für Hauptrechtsideale nennen wir $M M H R$-Ringe. Ein $M H U$-Ring bedeutet aber stets einen Ring mit Minimalbedingung für die durch ein Element erzeugten Unterringe.

Bekanntlich wird ein Ring $A$ im von Neumannschen Sinne regulär genannt, wenn es zu jedem Element $a \in A$ ein $x \in A$ mit $a=a x a$ gibt. Ist ferner $A$ ein beliebiger Ring mit einem idempotenten Element $e$, so wird das Hauptrechtsideal $(e)_{r}=e A$ von $A$,,regelmäßig” genannt, wenn der Unterring $Q_{e}=(1-e) A e A(1-e)$ nilpotent ist. Bezeichnet $I$ den Ring der ganzen rationalen Zahlen, so ist $1 \in J$ im Falle $1 \notin A$ ein Operator mit $(1-e) A e A(1-e)=$ $=\{a e b-e a e b-a e b e+e a e b e \mid a, b \in A\}$. Der Unterring $Q_{e}$ ist ein Quasiideal im Sinne von O. Steinfeld [23]. Bezeichnet $e_{i j}$ die Basiselemente des vollen Matrizenringes $A=(I /(2))_{2}(i, j=1,2)$, so ist $e_{11} A$ wegen $e_{22} \in Q_{e_{11}}$ kein regelmäßiges Hauptrechtsideal. Ist aber $A$ kein Nilring und ist $A$ entweder

1 Diese Arbeit ist ein Teil der Dissertation (1959) des Verfassers. Wir verweisen noch auf [26] und auf die Note [6], die voneinander unabhängig zustande gekommen sind. Vgl. noch F. Szász, A főjobbideálokra nézve minimum-feltételü gyürük, MTA Mat. és Fiz. Oszt. Közl., 11 (1961), S. 135-177, was eine abgekürzte Version der Kandidatsdissertation ist. 
ein $M M H R$-Ring oder ein Artinscher Ring, so ist $e A$ mit jedem Hauptidempotent $e$ regelmäBig. Wiederum ist $e A$ in einem beliebigen Ring $A$ regelmäßig, wenn $e\left(=e^{2}\right)$ zum Zentrum von $A$ gehört. Ist nun $\varphi$ ein Ringhomomorphismus von $A$ in $B$, so ist mit $e A$ auch $e \varphi \cdot A \varphi$ ebenfalls regelmäßig.

Ist $E$ der volle Endomorphismenring einer beliebigen Abelschen Gruppe $M$, und $A=A^{(0)}$ ein Unterring von $E$, so sei $A^{(n+1)}$ der Zentralisator von $A^{(n)}$ bezüglich $E$ für jede natürliche Zahl $n$. Es gelten immer $A^{(2 k-1)}=A^{(1)}$ und $A^{(2 k)}=A^{(2)}$ für alle $k \geqq 1$. Der kritische Fall ist im allgemeinen $k=0$. Ist nun $A$ die ringtheoretische direkte Summe beliebig vieler einfacher Artinscher Ringe, so gilt auch $A^{(0)}=A^{(2)}$.

Ein $A$-Rechtsmodul $M$ mit $M A=M$ ist perfekt. Ein vollständig reduzibler $A$-Faktormodul $M / K$ von $M$ wird ,,ausgezeichnet" genannt, wenn jeder $A$-Homomorphismus eines beliebigen vollständig reduziblen $A$-Faktormoduls $M / L$ auf $M / K$ ein Isomorphismus ist. Wir bezeichnen die Menge $\left\{x \mid x \in M, x \cdot J^{a}=0\right\}$ mit $K_{c}(M)$ für einen $A$-Rechtsmodul $M$ und für jede Ordnungszahl $\alpha$, wobei $J$ das Radikal von $A$ ist.

Nun werden wir die Ergebnisse der vorliegenden Arbeit zusammenfassen.

Im $\S 2$ werden das Noethersche und das Wedderburn-Artinsche Kriterium verallgemeinert, ferner Eindeutigkeitsfragen erörtert, bzw. spezielle halbeinfache $M H R$-Ringe charakterisiert. Es werden auch die subdirekten Darstellungen von $M H I$-Ringen, und eine Verallgemeinerung eines Kriteriums von A. KERTÉsz betrachtet, das die halbeinfachen Artinschen Ringe durch eine Folge von äquivalenten Bedingungen bestimmt hat. Wir verallgemeinern auch ein Harada-Kovácssches Kriterium über die regulären Ringe. Hiernach und nach [26] ist jeder halbeinfache $M H R$-Ring regulär. Wir haben aber e inen regulären Ring explizit gegeben, der kein (halbeinfacher) $M H R$-Ring ist

Im $\S 3$ werden die additiven Gruppen der $M H R$-Ringe, insbesondere der $M H R$-Ringe mit Einselement, der $M H R$-Radikalringe bzw. der primitiven $M H R$-Ringe betrachtet. In einem nilpotenten $M H_{1} R$-Ring gilt die Minimalbedingung auch für die in Hauptrechtsidealen liegenden additiven Untergruppen, und jeder Unterring ist ebenfalls ein $M H_{1} R$-Ring.

Im $\S 4$ untersuchen wir die Struktur einer Abelschen Gruppe $M$ mit (vollem) $M H R$-Endomorphismenring $E$. Ist nun der volle Endomorphismenring $E$ von $M$ beliebig, und $A$ ein einfacher $M H R$-Unterring von $E$, so kann nach einem expliziten Beispiel auch $(A=) A^{(0)} \neq A^{(2)}$ gelten, d. h. der Zentralisator $A^{(2)}$ des Zentralisators $A^{(1)}$ von $A$ bezüglich $E$ ist von $A$ verschieden. Wir werden auch die Operatorenmoduln und die Loewyschen Systeme über MHR-Ringe erörtern.

$\S 5$ untersucht die Zusammenhänge von verschiedenen Typen der Radikale von $M H R$-Ringen. In beliebigen $M H R$-Ringen stimmen das Baer- 
McCoysche Radikal $B$, das Levitzkische Radikal $K$, das obere Nilradikal $N$ und das Jacobsonsche Radikal $J$ überein. Hat der $M H R$-Ring ein Rechtseinselement, so stimmen auch das Fuchssche Radikal $Z$ und das BrownMcCoysche Radikal $G$ mit dem Jacobsonschen Radikal $J$ überein. Im allgemeinen gelten in $M H R$-Ringen $Z \supseteq J$ und $G \supseteq J$, und es können durch spezielle Beispiele auch $Z \neq J$ und $G \neq J$ bestätigt werden.

Im $\S 6$ betrachten wir die $M M H R$-Ringe und die regelmäßigen Hauptrechtsideale des Sockels eines Ringes. Hiernach ist jedes Rechtsideal $R$ eines regelmäßigen Hauptrechtsideales, als Ringes, des Sockels $A_{1}$ von $A$ ebenfalls ein Rechtsideal in $A$, und es gilt noch eine Folge der Behauptungen, insbesondere eine relative Charakterisierung bezüglich der regelmäßigen Hauptrechtsideale des Sockels eines Ringes.

Im $\S 7$ werden die $M H U$-Ringe erörtert. Es gibt einen $M H U$-Ring, der kein $M H R$-Ring ist, und einen $M H R$-Ring, der kein $M H U$-Ring ist. Ist nun $A$ ein $M H U$-Nilring, der auch ein $M H R$-Ring ist, so bilden die Elemente von $A$ bezüglich der Verknüpfung $x \cdot y=x+y-x y$ eine periodische verallgemeinerte auflösbare Gruppe $G$, die das direkte Produkt ihrer Sylowschen $p$-Untergruppen ist. Die Bedingung, daB jeder endlich erzeugbare echte Unterring von $A$ ein Hauptrechtsideal von $A$ ist, ist hinreichend aber nicht notwendig dafür, daß ein $M H R$-Ring auch ein $M H U$-Ring sei, bzw., daß ein $M H U$-Ring auch ein $M H R$-Ring sei (vgl. Satz 3 von [25]). ${ }^{2}$

\section{$\S$ 2. Über halbeinfache $M H R$-Ringe}

Wir haben schon in unserer Arbeit [26] gezeigt, daß jeder halbeinfache $M H R$-Ring $A$, als ein $A$-Rechtsmodul, vollständig reduzibel ist. Diese Ringe $A$ sind auch als zweiseitige $(A, A)$-Doppelmoduln vollständig reduzibel. Es gilt nun die folgende Behauptung als eine Umkehrung und Ergänzung dieser Tatsachen:

2. 1. Ist ein Ring $A$ die direkte Summe seiner idempotenten minimalen Rechtsideale, so ist A ein halbeinfacher MHR-Ring. Ein Ring A ist genau dann ein halbeinfacher MHR-Ring, wenn $A$ in die ringtheoretische direkte Summe von einfachen MHR-Ringen zerlegt werden kann. Diese letztere Zerlegung ist bis auf Isomorphie und Permutation eindeutig.

Nach [26] ist nämlich $A$, als die direkte Summe idempotenter minimaler Rechtsideale, auch als ein $(A, A)$-Doppelmodul vollständig reduzibel. Daher ist das Radikal $J$ ein direkter Summand von $A$, folglich $J=0$, denn

2 Vgl. F. SzÁsz, Die Ringe, deren endlich erzeugbare echte Unterringe Hauptrechtsideale sind, Acta Math. Acad. Sci. Hung., 13 (1962) (im Erscheinen), und die Kandidatsdissertation des Verfassers. 
aus $e^{2}=e \in J$ erhält man $e=0$. Die weitere Behauptungen folgen aus dem Kapitel III von [13].

SATz 2. 2. Jeder MHR-Ring A ohne nilpotente Elemente $(\neq 0)$ ist die ringtheoretische direkte Summe von Schiefkörpern (GERTSCHIKoff [11]). Jeder nullteilerfreie MHR-Ring ist ein Schiefkörper. Jeder kommutative halbeinfache MHR-Ring ist die ringtheoretische direkte Summe von Körpern. Jeder bireguläre [13] MHR-Ring ist die ringtheoretische direkte Summe von vollen Matrizenringen über Schiefkörpern.

BEwEIS. Jeder MHR-Ring $A$ ohne nilpotente Elemente $(\neq 0)$ ist nämlich halbeinfach, denn das Radikal ist ein Nilideal. Nach dem Satz von LiToff (Kapitel IV von [13]) läßt sich aber jeder endlich erzeugbare Unterring von $A$ in einen solchen Unterring von $A$ einbetten, der einem vollen Matrizenring über einem Schiefkörper isomorph ist, und der im allgemeinen nilpotente Elemente $(\neq 0)$ hat. Hiernach ist jede zweiseitige einfache direkte Komponente von $A$ ein Schiefkörper.

Ist nun insbesondere $A$ nullteilerfrei, so ist $A$ selbst ein Schiefkörper.

Da jeder kommutative einfache Ring ein Körper ist, und jedes (zweiseitige) Hauptideal eines biregulären Ringes ein Einselement hat, gelten auch die weiteren Behauptungen im Satz 2.2.

Bemerkung. Der Satz von Szele [27] ist eine Folgerung sowohl des Wedderburn-Artinschen Struktursatzes als auch unserer Resultate über $M H R-$ Ringe. Dagegen kann auch ein kürzer elementarer Beweis des Szeleschen Satzes über die Ringe ohne echte Rechtsideale gegeben werden (vgl. Lemma 1 von [24]).

Satz 2. 3. Jeder halbeinfache $M H R$-Ring ist ein $M H_{1} I$-Ring. Ein MHIRing $A$ ist dann und nur dann eine ringtheoretische subdirekte Summe einfacher Ringe $E_{\alpha}^{\prime}(\alpha \in \Omega)$, wenn $A$ die ringtheoretische direkte Summe der Ringe $E_{\alpha}\left(\cong E_{\alpha}^{\prime}\right)$ ist. Dann ist $A$ auch ein $M H_{1} I-R i n g$.

BEwEIS. Ein halbeinfacher $M H R$-Ring ist zweiseitig vollständig reduzibel, also ist er ein $M H_{1} I$-Ring.

Es sei nun $A$ ein $M H I$-Ring, der eine subdirekte Summe einfacher Ringe $E_{\alpha}^{\prime}(\alpha \in \Omega)$ ist. Dann existieren maximale Ideale $B_{\alpha}$ mit $A / B_{\alpha} \cong E_{\alpha}^{\prime}$, $\bigcap_{a \in \Omega} B_{a}=0, A^{2}=B_{\alpha}+A$. Wir zeigen, daß jedes Hauptideal $(a)$ von $A$, als ein $(A, A)$-Doppelmodul, vollständig reduzibel ist. Es sei nämlich $C_{\alpha}=(\alpha) \cap B_{\alpha}$. Dann können die Ideale $C_{a}$ mit $C_{\alpha}=(a)$ weggelassen werden, und $(a)$ ist eine subdirekte Summe der übrigbleibenden einfachen Ringe $(a) / C_{a}\left(\simeq A / B_{\alpha}\right.$; $\left.a \notin B_{a}\right)$. Ist nun $(m)$ ein beliebiges minimales Ideal von $A$ in $(a)$, so existiert ein Ideal $C_{\alpha}$ mit $(m) \ddagger C_{\alpha}$, also mit $(a)=(m) \oplus C_{\alpha}$. Hiernach ist das endo- 
morphe Bild $C_{a}$ von $(a)$ ebenfalls ein Hauptideal. Ist nun $\left(m_{1}\right)$ ein minimales Ideal von $A$ in $C_{\alpha}$, so gilt $C_{\alpha}=\left(m_{1}\right) \oplus C_{\beta}$ mit einem Hauptideal $C_{\beta}$ von $A$. Da aber $A$ ein $M H I$-Ring ist, besteht $(a)=(m) \oplus\left(m_{1}\right) \oplus \cdots \oplus\left(m_{k}\right)$ mit einem geeigneten Index $k$, denn $(a) \supset C_{\alpha} \supset C_{\beta} \supset \cdots$ muß abbrechen. Deshalb ist aber auch $A=\bigcup_{a \in A}(a)$, als ein $(A, A)$-Doppelmodul, vollständig reduzibel, w. z. b. w.

Herr Dr. A. KERTÉsz hat in [15] und [16] bewiesen, daß eine Folge untereinander äquivalenter Bedingungen unter den Ringen mit Linkseinselement genau die Artinschen halbeinfachen Ringe charakterisiert. Diese Bedingungen bestimmen aber genau die halbeinfachen $M H R$-Ringe in der größeren Klasse der Ringe ohne Rechtsannullatoren $(\neq 0)$. Ist nun $A^{\prime}=A$, wenn $A$ ein Einselement hat, und sonst $A^{\prime}$ die Dorrohsche Erweiterung von $A$ mit Einselement, so gilt der

SATz 2. 4. Für einen Ring A ohne Rechtsannullatoren $(\neq 0)$ sind die folgenden Bedingungen untereinander äquivalent:

a) A ist ein halbeinfacher MHR-Ring;

b) A ist die direkte Summe idempotenter minimaler Rechtsideale;

c) A ist ein MHR-Ring und eine subdirekte Summe von Ringen, die zu idempotenten minimalen Rechtsidealen von A A-isomorph sind;

d) A ist ein MHR-Ring, in dem der Durchschnitt gewisser modularer ([13]) maximaler Rechtsideale (0), ist;

e) A stimmt mit seinem Sockel $A_{1}$ überein;

f) der Rechtsannullator in $A^{\prime}$ jedes Elementes von $A$ ist der Durchschnitt endlich vieler modularer maximaler Rechtsideale von $A^{\prime}$;

g) es gibt zu jedem Rechtsideal $R$ von $A$ ein Rechtsideal $S$ von $A$ mit $A=R+S, R \cap S=0$;

h) existiert die direkte Summe $R^{*}=\sum_{\alpha \in \Omega} \oplus R_{\alpha}$ für eine möglichst maximale Menge der minimalen Rechtsideale $R_{\alpha}(\alpha \in \Omega)$, so besteht $A==R^{*}$.

BEweIs. Gilt $A=A_{1}$ für den Sockel (d. h. Rechtssockel) $A_{1}$ von $A$, so ist $A$ wegen $A_{1} \cdot J=0$ genau dann halbeinfach, wenn $A$ keinen Rechtsannullator $(\neq 0)$ besitzt. Betrachten wir nun $A^{+}$als einen $A^{\prime}$-Rechtsmodul. Der Beweis folgt nun aus dem Kertészschen Hauptsatze in [16].

Bemerkungen. Ist jedes Rechtsideal $R$ eines Ringes $A$, als eines $A$ Rechtsmoduls, ein rechtsseitiger direkter Summand, so gilt $A=B \oplus C$, wobei $\oplus$ eine ringtheoretische direkte Summe, $B$ ein halbeinfacher $M H R$-Ring und $C$ ein Zeroring mit additiver elementarer Abelscher Gruppe ist. (Die additive Ordnung $\mathrm{O}^{+}(c)$ jedes Elementes $c$ von $C$ ist eine quadratfreie Zahl.) - Ist insbesondere jedes Rechtsideal $R$ eines Ringes $A$, als eines $(A, A)$-Doppel- 
moduls, ein zweiseitiger direkter Summand, so gilt $A=B \oplus C$, wobei $B$ die ringtheoretische direkte Summe von Schiefkörpern, und $C$ ein Zeroring mit elementarer Abelscher additiver Gruppe ist.

SATz 2.5. Ein Ring A ist dann und nur dann die ringtheoretische direkte Summe von Schiefkörpern, wenn jedes in einem Hauptrechtsideale liegende Rechtsideal $R$ von $A$ ein Rechtseinselement e besitzt.

BEweIs. Es seien also $e$ und $e_{1}$ Rechtseinselemente von $R \subseteq(a)_{r}$ bzw. von $R_{1}=(1-e) R\left(\subseteq(a)_{r}\right)$. Da $R_{1}^{2}=(1-e) R(1-e) R=0$ ist, folgt aus der Halbeinfachheit von $A$ auch $R_{1}=0$. Dies bedeutet aber, daß $e$ gleichzeitig auch ein Linkseinselement von $R$ ist. Nach unserem Satz 4 in [26] ist aber ein beliebiger Ring $B$ dann und nur dann ein halbeinfacher $M H R$-Ring, wenn jedes in einem Hauptrechtsideale liegende Rechtsideal $R$ von $B$ ein Linkseinselement besitzt. Hiernach ist also $\operatorname{der} \operatorname{Ring} A$ ein halbeinfacher $M H R$-Ring. Gilt nun $e_{1} A \cap e_{2} A=0\left(e_{i}^{2}==e_{i}\right)$, so hat $e_{1} A+e_{z} A$ ebenfalls ein (zweiseitiges) Einselement, und die Komponenten dieses Einselementes können als $e_{1}$ und $e_{2}$ mit $e_{1} e_{2}=e_{2} e_{1}=0$ gewählt werden. Dann ist jedes Rechtsideal von $A$ ein Ideal, und somit ist $A$ die ringtheoretische direkte Summe von Schiefkörpern. Die Umkehrung ist trivial.

SATz 2.6. Ein beliebiger Ring $A$ ist dann und nur dann regulär im von Neumannschen Sinne, wenn jedes Hauptrechtsideal von A ein Linkseinselement hat. Ein beliebiger Ring $A$ ist dann und nur dann regulär, wenn $(a)_{r}(a)_{l}=(a)_{r} \cap(a)_{l}$ für jedes Element $a \in A$ gilt. (Vgl. S. Lajos und K. IsÉKI bezüglich Halbgruppen.) Jeder halbeinfache MHR-Ring ist regulär.

BeweIs. Der Ring $A$ besitzt nicht notwendig ein Einselement. (Wir verweisen bezüglich regulärer Ringe ohne Einselement auf McCoy's Buch.) Hiernach sei $e=n a+a b \quad(n \in I ; a, b \in A)$ ein Linkseinselement von $(a)$. Dann gilt aber $a=a c$ mit $c=n a+b a$, also $a=e a c=a(n+b) a(n+b) a$. Dies bedeutet die Regularität von A. Umgekehrt folgt nun aus $a=a d a$ $(d \in A)$ auch $a d(n a+a x)=n a+a x$, also die Existenz eines Linkseinselementes $e=a d$ in $(a)_{r}$.

Gilt $(a)_{r} \cdot(a)_{l}=(a)_{r} \cap(a)_{l}$ für jedes $a \in A$, so ergibt sich aus $a=\left(n_{\mathrm{i}} a+a b_{1}\right)$. $\cdot\left(b_{2} a+n_{2} a\right) \quad\left(n_{i} \in I ; b_{i} \in A\right)$ durch eine wiederholte Einsetzung $a=a\left[\left(n_{1}+b_{1}\right)\right.$. $\left.\cdot\left(b_{2} a+n_{2} a\right)\left(n_{1}+b_{1}\right)\left(n_{2}+b_{2}\right)\right] a$, also die Regularität von $A$. Umgekehrt genügt es, in regulären Ringen $(a)_{r} \cap(a)_{l} \subseteq(a)_{r} \cdot(a)_{l}$ zu beweisen. Jedes Element des Durchschnittes hat die Form $b=a y=z a(y, z \in A)$. Gilt nun $a=a x a$, so ist auch $b=z a=z a x a=a y x a \in(a)_{r} \cdot(a)_{l}$, w. z. b. w.

Die letzte Behauptung des Satzes 2.6 ist eine Folgerung der obigen Verallgemeinerung des von Neumannschen Kriteriums bezüglich regulärer Ringe, und die unserer Arbeit [26]. 
Also ist jeder halbeinfache $M H R$-Ring regulär, und bekanntlich ist jeder reguläre Ring halbeinfach (im Jacobsonschen Sinne). Das folgende Beispiel zeigt aber die Existenz eines regulären Ringes, der kein $M H R$ Ring ist.

Beispiel 2.7. Es sei $A$ die komplette direkte Summe unendlich vieler regulärer Ringe $A_{1}, A_{2}, \ldots, A_{n}, \ldots$ usw. Es seien $b_{1}=\left\langle a_{11}, a_{12}, \ldots, a_{1 n}, \ldots\right\rangle$ bzw. $b_{k}=\left\langle 0,0, \ldots, 0, a_{k k}, a_{k, k+1}, \ldots\right\rangle$ die Elemente von $A$ mit beliebigen $a_{1 n}\left(\neq 0, \in A_{1}\right)$ und mit $a_{k-1, n} \cdot a_{k n}=a_{k-1, n}\left(\in A_{n}\right)$. Dann ist $\left(b_{1}\right)_{r} \supset\left(b_{1} b_{2}\right)_{r} \supset$ $\supset\left(b_{1} b_{2} b_{3}\right)_{r} \supset \ldots$ eine unendliche absteigende Kette der Hauptrechtsideale, obwohl $A$ offenbar regulär ist. Es kann in $A$ auch ein in einem Hauptrechtsideale liegendes Rechtsideal ohne Linkseinselemente explizit gegeben werden: $R^{*}=\sum_{j=1}^{\infty} \oplus\left(e_{j}-e_{j+1}\right) e_{j} A$, wobei $\quad\left(b_{1} \cdot b_{2} \ldots b_{j}\right)_{r}=e_{j} A\left(e_{j}^{2}=e_{j}\right)$ ist.

Zum Schluß erwähnen wir eine Charakterisierung der $M H_{1} R$-Ringe.

SATZ 2. 8. Ein Ring $A$ ist dann und nur dann ein $M H_{1} R$-Ring, wenn die Minimalbedingung für die in Hauptrechtsidealen liegenden Nilrechtsideale von A gilt, und jedes in einem Hauptrechtsideale liegende Rechtsideal $R$ von $A$ die Gestalt $R=e A \oplus R_{1}$ hat, wobei $e^{2}=e$ und $R_{1}$ ein Nilrechtsideal von A ist.

Bemerkung 2.9. Da jeder nullteilerfreie einfache $M H R$-Ring ein Körper ist, scheint es uns sehr merkwürdig, alle nullteilerfreien einfachen Ringe zu bestimmen. Ist ein nullteilerfreier einfacher Ring kein Schiefkörper, so ist er aber deswegen kein $M H R$-Ring. Es kann aber auch ein solcher Ring ergeben werden. Man nimmt nämlich einen nullteilerfreien Ring $A$, der sich in keinen Schiefkörper aber in einen einfachen nullteilerfreien Ring $E$ einbetten läßt (Malczew; Conn). Dann hat $E$ sicher die obigen Eigenschaften. Wir wissen bisher noch nicht, ob was ein Kriterium dafür ist, daß ein $M H R$-Ring in einen einfachen $M H R$-Ring eingebettet werden kann. Man wünscht ebenfalls eine notwendige und hinreichende Bedingung dafür $\mathrm{zu}$ suchen und finden, $\mathrm{da} B$ in einer subdirekten Summe $A$ beliebiger einfacher Ringe $E_{a}$ die Ringe $E_{a}$ bis auf Isomorphie und Permutation durch den ursprünglichen Ring $A$ eindeutig bestimmt seien. Man möchte auch die solchen Ringe explizit bestimmen, deren jedes in einem Hauptrechtsideale liegende Linksideal ein Rechtseinselement (bzw. Linkseinselement) hat. Die ringtheoretischen direkten Summen von Schiefkörpern sind nämlich sicher solche Ringe. Wir wissen bisher ebenfalls auch noch nicht, ob es einen Ring gibt, der die direkte Summe von $m$ idempotenten minimalen Rechtsidealen und die von $\pi$ idempotenten minimalen Linksidealen ist, wobei $m$ und $n$ zwei verschiedene unendliche Mächtigkeiten sind. 


\section{§ 3. Die additive Gruppe der $M H R$-Ringe}

Die Methoden von L. Fuchs-T. Szele über die additive Gruppe der Artinschen Ringe, die später von L. Fuchs weiterentwickelt wurden [7], können auch auf die Untersuchung der additiven Gruppe der MHR-Ringe angewandt und verallgemeinert werden.

Satz 3.1. Eine Abelsche Gruppe $A^{+}$ist dann und nur dann die additive Gruppe eines $M H R$-Ringes, wenn $A^{+}=B \oplus C$ gilt, wobei $B$ vollständig und $C$ reduziert periodisch sind. Existiert eine Prüfersche Gruppe $Z\left(p^{\infty}\right)$ in $A^{+}$, so liegt $Z\left(p^{\infty}\right)$ im zweiseitigen Annulator des MHR-Ringes $A$.

Bewers. Ist $A$ ein $M H R$-Ring und $B$ die maximale vollständige Untergruppe von $A^{+}$, so gilt $A^{+}=B \oplus C$ mit reduzierter $C$. Ist nun $(\mathrm{ma})_{r}$ $\left(=m(a)_{r}\right)$ ein minimales Hauptrechtsideal unter den Hauptrechtsidealen $(n a)_{r}(n \in I)$, so gilt $m a \in B$. Daher ist $C$ periodisch wegen $B \cap C=0$. Umgekehrt habe $A^{+}$die erwähnte Struktur, und es sei $B=B_{1} \oplus B_{2}$ mit torsionfreier $B_{1}$ und mit periodischer $B_{2}$. Definiert man nun über $B_{1}$ einen kommutativen Körper [7] und sei $B_{2} A=A B_{2}=C A=A C=0$, so entsteht über $A^{+}$ein spezieller $M H R$-Ring.

Ist ferner $Z\left(p^{\infty}\right) \subseteq A^{+}, a \in Z\left(p^{\infty}\right), \quad O(a)=p^{k}, A^{+}=B \oplus C$ mit den vorigen Bezeichnungen bezüglich des $M H R$-Ringes $A$, so gilt $x=b+c(x \in A)$ mit $b \in B, c \in C, O(c)=l$. Die Gleichungen $l u=a$ und $p^{k} v=b$ sind in $A^{+}$ lösbar, und es gilt $a x=a b+a c=a\left(p^{k} v\right)+(l u) c=\left(p^{k} a\right) v+u(l c)=0$, also $Z\left(p^{\infty}\right) \cdot A=0$. Ganz ähnlich ergibt sich auch $A \cdot Z\left(p^{\infty}\right)=0$.

SATZ 3. 2. $A^{+}$ist dann und nur dann die additive Gruppe eines $M H R-$ Ringes mit Einselement, wenn $A^{+}=B \oplus C$ gilt, wobei $B$ torsionsfrei vollständig und $C$ beschränkt periodisch sind. $A^{+}$ist genau dann die additive Gruppe eines primitiven $M H R$-Ringes, wenn $A^{+}$entweder eine torsionsfreie vollständige Gruppe oder eine elementare Abelsche p-Gruppe ist.

Bewers. Ist $A$ ein $M H R$-Ring mit Einselement, so gilt $Z\left(p^{\infty}\right) \ddagger A^{+}$. Die maximale vollständige Untergruppe $B$ von $A^{+}$ist also torsionsfrei, und der maximale periodische Unterring $C$ von $A$, als ein ringtheoretischer direkter Summand von $A$, ist ein endomorphes Bild des Ringes $A$. Also hat der Ring $C$ ein Einselement $e$ mit $O(e)=m$. Hiernach gilt aber $m C=0$. Besteht nun umgekehrt $A^{+}=B \oplus C$ mit torsionsfreier vollständiger $B$ und mit beschränkter periodischer $C$, so konstruieren wir auf $B$ einen kommutativen Körper [7]. Ferner ist $C$ die direkte Summe von Gruppen $Z\left(p_{m}^{k_{i}}\right)$ für gewisse Primzahlen $p_{m}$ und Exponenten $k_{i}$. Wir sammeln nun die Summanden $Z\left(p_{m}^{k_{i}}\right)$, die zu einem festen Paar $\left(p_{m}, k_{i}\right)$ gehören, in eine Summe $D_{m, k}$ zusammen. Nach einem wichtigen Lemma von L. Fuchs ([7], S. 281) kann auf jede Gruppe 
$D_{m, k}$ ein kommutativer Ring mit Einselement aufgebaut werden, dessen sämtliche verschiedene Ideale $0, p_{m} D_{m, k}, p_{m}^{2} D_{m, k}, \ldots, p_{m}^{k-1} D_{m, k} \neq 0$ sind. Da nur endlich viele verschiedene $D_{m, k}$ existieren, entsteht mit den Definitionen $B D_{m, k}=D_{m, k} B=0$ und $D_{m, k} \cdot D_{n, l}=D_{n, l} D_{m, k}=\delta_{m n} \delta_{k l} D_{m, k} \quad$ offenbar ein $M H R$-Ring $A$ über $A^{+}$.

Jeder primitive $M H R$-Ring ist nach [26] einfach. Gilt nun $p A=A$ für jede Primzahl $p$, so ist $A^{+}$vollständig, und zwar torsionsfrei nach der letzten Behauptung des Satzes 3.1. Im Falle $p A \neq A$ ist aber $p A=0$, und hiernach ist $A^{+}$eine elementare Abelsche $p$-Gruppe. Umgekehrt können auf die so bekommenen Gruppen kommutative Körpern [7] aufgebaut werden, w. z. b. w.

SATZ 3. 3. Die Abelsche Gruppe $A^{+}$ist dann und nur dann die additive Gruppe eines MHR-Radikalringes, wenn $A^{+}$periodisch ist. Ist insbesondere $A$ ein nilpotenter $M H_{1} R$-Ring, so gilt in $A$ die Minimalbedingung auch für die in Hauptrechtsidealen liegenden additiven Untergruppen von $A^{+}$,

BeweIs. Es sei $A^{+}$die additive Gruppe eines $M H R$-Radikalringes $A$. Dann ist $A$ ein Nilring nach dem Satz 1 unserer Arbeit [26]. Wäre nun $x(\neq 0, \in A)$ ein Element der Ordnung $O^{+}(x)=0$, so würde $\left(2^{k} x\right)_{r}=\left(2^{k+1} x\right)_{r}$ mit einem Exponenten $k$ bestehen. Dann gilt aber mit der Bezeichnung $y=2^{k} x$ eine Gleichung $y=n(2 y)+(2 y) z$, folglich auch $(2 n-1)^{m} y=$ $=(2 n-1)^{m-1}(-2 z) y=\cdots=(-2 z)^{m} y=0 \quad\left(n \in I, z \in A, z^{m}=0\right)$. Da aber $O(y)=0$ gilt, und der Ring $I$ der ganzen Zahlen nulteilerfrei ist, gelten $2 n-1=0$ und $n=1 / 2 \in I$, was unmöglich ist. Hiernach ist $A^{+}$tatsächlich periodisch. Umgekehrt kann auf jede periodische additive Gruppe ein Zeroring als ein spezieller $M H R$-Radikalring aufgebaut werden.

Es sei nun $A$ ein nilpotenter $M H_{1} R$-Ring, $A^{n}=0\left(A^{n-1} \neq 0\right)$ und $L_{k}$ $(k=1,2,3, \ldots, n-1)$ der Linksannullator von $A^{k}$ in $A$. Dann ist $0 \subset L_{1} \subset$ $\subset L_{2} \subset \cdots \subset L_{n-1}=A$. Es sei ferner $G_{1} \supset G_{2} \supset G_{3} \supset \ldots$ eine unendliche absteigende Kette von in einem Hauptrechtsideale $(a)_{r}$, von $A$ liegenden additiven Untergruppen von $A^{+}$, und $a \in L_{t}$ mit $t \in I, 1 \leqq t \leqq n-1$. Ist nun $R_{i}=G_{i}+G_{i} A$, so gelten $R_{i} \subseteq(a)_{r}$ und $R_{i} A \subseteq R_{i}$. Da aber $A$ ein $M H_{1} R$-Ring ist, so existiert ein Index $i_{1}$ mit $R_{i_{1}+1}=R_{i_{1}+2}=\ldots$, also auch mit $G_{i_{1}+1}+L_{t-1}=$ $=G_{i_{1}+2}+L_{t-1}=\ldots$ wegen $G_{i} A \subseteq L_{t} A \subseteq L_{t-1}$. Aus $x \in L_{t} A$ erhält man nämlich $x A_{t-1}$, folglich auch $x \in L_{t-1}$ und $L_{t-1} \supseteq L_{t} A$. Da aber der Verband der Untergruppen von $A^{+}$modular ist, gilt $G_{i_{1}+1} \cap L_{t-1} \supset G_{i_{1}+2} \cap L_{t-1} \supset \ldots$ Nach endlich vielen Schritten gewinnen wir die Existenz eines Indexes $i_{t-1}$ mit $G_{i_{t-1}+1}+L_{1}=G_{i_{t-1}+2}+L_{1}=\ldots$, folglich auch mit $G_{i_{t-1}+1} \cap L_{1} \supset G_{i_{t-1}+2} \cap L_{1} \supset \ldots$ Diese unendliche Kette besteht wegen $L_{1} A=0$ aus in $(a)_{r}$. liegenden Rechtsidealen von $A$, und da $A$ ein $M H_{1} R$-Ring ist, muß auch die Kette $G_{1} \supset G_{2} \supset G_{3} \supset \ldots$ nach endlich vielen Schritten abbrechen, w. z. b. w. 
Bemerkung 3.4. Es kann mit einer ähnlichen Methode gezeigt werden, daß jeder Unterring eines nilpotenten $M H_{1} R$-Ringes ebenfalls ein $M H_{1} R$ Ring ist. Gilt ferner die Maximalbedingung für die in einem Hauptrechtsideale liegenden Rechtsideale eines nilpotenten $M H_{1} R$-Ringes $A$, so gilt die Maximalbedingung auch für die in einem Hauptrechtsideale liegenden additiven Untergruppen von $A^{+}$. Wir wissen aber noch nicht, ob es einen $M H R-$ Radikalring (bzw. einen nilpotenten $M H R$-Ring bzw. einen $M H_{1} R$-Nilring) gibt, dessen ein Unterring $S$ die entsprechende Kettenbedingung nicht erbt. Auch das Problem der Existenz eines MHR-Nilringes ohne die Minimalbedingung für Hauptlinksideale bildet für uns bisher eine offene Frage. Wir wissen ferner ebenfalls noch nicht die Lösung von zwei wichtigen Problemen bezüglich Abelscher Gruppen: 1. Was ist eine notwendige und hinreichende Bedingung dafür, daß die Abelsche Gruppe $G$ einen vollen Endomorphismenring mit Minimalbedingung für zweiseitige Hauptideale besitze? 2. Was ist ein Kriterium dafür, daß auf eine Abelsche Gruppe $G$ wenigstens ein $M H R$-Ring mit Maximalbedingung für Hauptrechtsideale (kurz: $M M H R-$ Ring) aufgebaut werden kann?

\section{§ 4. Die $M H R$-Ringe als Operatorenbereiche}

Wir werden die Operatoren und die Endomorphismen einer Abelschen Gruppe $M$ stets rechtsseitig schreiben. Offensichtlich erhält man nun zwei verschiedene Problemkreise, wenn man eine Eigenschaft erstens für den Verband der Linksideale von $E$, zweitens für den Verband der Rechtsideale von $E$ erfordert, wobei $E$ den vollen Endomorphismenring von $M$ bezeichnet.

SATz 4.1. Ist $E$ der volle Endomorphismenring einer Abelschen Gruppe $M$, so sind die folgenden Bedingungen untereinander äquivalent:

I. in E gilt die Minimalbedingung für Hauptrechtsideale;

II. in E gilt die Minimalbedingung für Hauptlinksideale;

III. in E gilt die Minimalbedingung für Rechtsideale;

IV. in E gilt die Minimalbedingung für Linksideale;

V. $M$ ist die direkte Summe einer endlichen Abelschen Gruppe und endlich vieler Exemplare $\mathfrak{R}$ der additiven Gruppe aller rationalen Zahlen.

Beweis. Aus I folgt V. Nehmen wir I an. Da $E$ ein Einselement $e$ hat, ist $n E$ für jede ganze Zahl $n$ ein Hauptrechtsideal in $E$. Ist nun $m E$ ein minimales Hauptrechtsideal unter den Rechtsidealen $n E$, so ist $m E^{+}$eine vollständige Gruppe, also gilt $k(m E)=m E$ für jedes $k(\in I, \neq 0)$. Dann ist aber $m M$ wegen $m M=m(M E)=M(m E)=M(k m E)=k m(M E)=k m M$ ebenfalls vollständig. Es gibt also eine Untergruppe $M_{0}$ von $M$ mit 
$M=m M \oplus M_{0}$ nach dem Satz von BAER, wobei $M_{0}$ offenbar eine $m$-beschränkte periodische Gruppe ist. Da $m e=k m x_{k}$ in $E$ für jedes $k(\neq 0, \in I)$ lösbar ist, gilt $Z\left(p^{\infty}\right) \nsubseteq M$. Folglich ist $m M$ torsionsfrei. Ferner ist $M_{0}$ die direkte Summe von zyklischen Gruppen wegen $m M_{0}=0$ und nach dem Satz von BAER. Ist nun $M=\sum_{i \geqq 1} \oplus M_{i}\left(M_{i} \neq 0\right)$ eine direkte Zerlegung von $M$, so bilden die direkten Summanden $S_{n}=\sum_{j \geqq n+1} \oplus M_{j}$ eine absteigende Kette, und

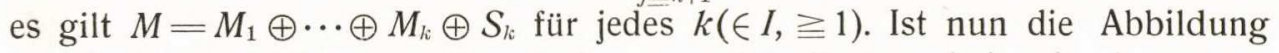
$a_{n} \in E$ die Projektion von $M$ auf $S_{n}$, so gilt offenbar $\left(a_{n}\right)_{r} \subseteq\left(a_{n-1}\right)_{r}$ wegen $a_{n-1} a_{n}=a_{n}$. Besteht aber $\left(a_{n}\right)_{r}=\left(a_{n-1}\right)_{r}$ im $M H R$-Ring $E$, so ist die Gleichung $a_{n} y_{n}=a_{n-1}$ in $E$ lösbar, und so ergibt sich $M_{n}=M_{n} a_{n-1}=$ $=M_{n} a_{n} y_{n}=0$ wegen $M_{n} a_{n}=0$. Dies bedeutet nun, daß $M$ die direkte Summe nur endlich vieler ihrer Untegruppen ist. Hiernach folgt aus I tatsächlich V.

Aus II folgt V. Der Beweis ist dem ,aus I folgt V', ähnlich. Man bestätigt aber $\left(a_{n}\right)_{l} \subseteq\left(a_{n-1}\right)_{l}$ wegen $a_{n} a_{n-1}=a_{n}$. Ist $E$ ein Ring mit Minimalbedingung für Hauptlinksideale (d. h. ein $M H L$-Ring), so hat $z_{n} a_{n}=a_{n-1}$ in $E$ Lösungen, wenn $n \geqq n_{0}$ ist. Aus $M_{n}=M_{n} a_{n-1}=M_{n} z_{n} a_{n} \subseteq M a_{n}=S_{n}$ folgt aber $M_{n}=0$ wegen $M_{n} \cap S_{n}=0$, und dies bedeutet wirklich das Bestehen von $\mathrm{V}$.

Aus $\mathrm{V}$ folgen III und IV. Im Falle $\mathrm{V}$ ist nämlich der volle Endomorphismenring $E_{1}$ der torsionsfreien vollständigen Gruppe $m M$ ein voller Matrizenring vom Typ $n \times n$ über dem rationalen Zahlkörper $K_{0}$. Der volle Endomorphismenring $E_{2}$ der endlichen Gruppe $M_{0}$ ist aber offenbar endlich. Da ferner die additive Gruppe $\Re$ aller rationalen Zahlen keine echte Untergruppe von endlichem (gruppentheoretischem) Index hat, so ist $\operatorname{Hom}\left(m M, M_{0}\right)=0$. Aus der Torsionsfreiheit von $m M$ folgt nun auch $\operatorname{Hom}\left(M_{0}, m M\right)=0$. Hiernach gewinnen wir eine ringtheoretische direkte Zerlegung $E=E_{1} \oplus E_{2}$. Also gilt in $E$ die Minimalbedingung sowohl für die Rechtsideale, als auch für die Linksideale.

Aus III folgt I. Dies ist trivial.

Aus IV folgt II. Dies ist ebenfalls trivial.

Das folgende Beispiel zeigt uns die Existenz einer Abelschen Gruppe $M$ derart, daß der Zentralisator $A^{(2)}$ des Zentralisators $A^{(1)}$ von einem einfachen $M H R$-Unterringe $A$ des vollen Endomorphismenringes $E$ von $M$ bezüglich $E$ von $A\left(=A^{(0)}\right)$ verschieden ist.

BeIspiel 4.2. Es sei nämlich $A$ der Ring aller $\aleph_{0} \times \aleph_{0}$ Matrizen mit endlich vielen Spalten- und Zeilenvektoren $(\neq 0)$ über dem rationalen Zahlkörper $K_{0}$. Bezeichne $e_{i j}$ die Matrizeneinheiten $(i, j=1,2, \ldots, n, \ldots)$ und $h_{k l}$ einen Homomorphismus mit $e_{i j} h_{k l}=\delta_{i k} e_{l j}$. Dann ist dieser $h_{m k}$ ein 
$A$-Homomorphismus wegen $\left(e_{i j} e_{k l}\right) h_{m n}=\left(e_{i j} h_{m n}\right) e_{k l}$ im vollständig reduziblen $A$-Rechtsmodul $A$, und es gelten $h_{m n} \in A^{(1)}, \sum_{i=1}^{\infty} h_{i i}=1, \quad h_{i j} h_{k l}=\delta_{j k} h_{i l}$, wenn wir den Ring $A$ mit dem Ring $\bar{A}$ aller Rechtsmultiplikationen $\bar{a}(a \in A)$ und $K_{0}$ mit dem Körper aller $A$-Endomorphismen von $\left(e_{11}\right)_{r}$ identifizieren. Es sei ferner $r_{i j}=\sum_{n=1}^{\infty} h_{n 1} r_{i j} h_{1 n} \quad\left(r_{i j} \in K_{0}\right), \quad b \in A^{(1)} \quad$ ein beliebiges Element, und $b_{i j}=h_{i i} b h_{i j}$. Dann gilt $b=\sum_{i, j}^{\infty} b_{i j}=\sum_{i, j}^{\infty} r_{i j} h_{i j}=\sum_{i, j}^{\infty} h_{i j} r_{i}$, wegen $h_{i j} r_{i j}=$ $h_{i 1} r_{i j} h_{1 j}=r_{i j} h_{i j}$. Ist nun $a_{1}=e_{1 i} h_{1 i} b_{i j} h_{j 1}\left(\in\left(e_{11}\right)_{r}\right)$, so ist $e_{1 i} x \rightarrow a_{1} x(x \in A)$ ein $A$-Endomorphismus von $\left(e_{11}\right)_{r}$, also gilt $h_{1 i} b_{i j} h_{j 1}=s_{i j} \in K_{0}$. Hiernach erhält man $b_{i j}=h_{i 1} s_{i j} h_{1 j}=s_{i j} h_{i j}=h_{i j} s_{i j}$ und $b=\sum_{i, j}^{\infty} s_{i j} h_{i j}$, wobei $s_{i j}$ nur für endlich viele $j$ von Null verschieden ist, denn $e_{k l} b$ muß nur aus endlich vielen Gliedern $(\neq 0)$ bestehen. Bezeichne nun $A^{*}$ den Ring aller formalen unendlichen Summen $a^{*}=\sum_{j}^{\prime} \sum_{i=1}^{\infty} r_{i j} e_{i j}$ mit $r_{i j}$ mit $r_{i j} \neq 0$ nur für endlich viele $j$. Ist ferner $M=A, \quad m \in M, a^{*} \in A^{*}, \quad b \in A^{(1)}$, so gilt $\left(m a^{*}\right) b=(m b) a^{*}$, folglich auch $A^{*} \subseteq A^{(2)}$. Also erhält man $A^{(0)} \neq A^{(2)}$ wegen $A^{(0)}=A=M=\bar{A} \subset A^{*}$, w. z. b. w.

SATZ 4.3. Ist A ein halbeinfacher MHR-Ring, so ist jeder perfekte A-Modul M vollständig reduzibel, und die Mächtigkeit der Menge aller A-nichtisomorphen irreduziblen A-Moduln ist gleich der Mächtigkeit von Menge aller einfachen Ideale von $A$.

BeweIS. Bekantlich ist jeder treue minimale $A$-Modul über einem primitiven Ring $A$ mit $\neq 0$ Sockel einem minimalen Rechtsideale $R$ von $A$ notwendig isomorph. Ähnlich ist jeder perfekte minimale $A$-Modul über einem halbeinfachen $M H R$-Ring $A$ einem minimalen Rechtsideale $R$ des Operatorenbereiches $A$-isomorph. - Ist nun $M(=M A)$ ein beliebiger $A$-Modul über einem halbeinfachen $M H R$-Ring $A$, so besteht $m=\Sigma m_{i} a_{i}$ mit $m_{i} a_{i} \neq 0\left(m_{i} \in M ; a_{i} \in A\right)$ und mit $a_{i}=\Sigma b_{i j}$, wobei $b_{i j}$ in einem minimalen Rechtsideale $R_{i j}$ von $A$ liegt. Ist nun $m_{i} b_{i j} \neq 0$, so ist $m_{i} R_{i j}(\neq 0)$ ein minimaler $A$-Untermodul von $M$, und somit ist $M$ vollständig reduzibel. Die letzte Behauptung folgt durch klassische Methoden.

Bemerkung. Das folgende Beispiel zeigt, daß es einen einfachen $M H R-$ Ring $A$ und einen $A$-Modul $M$ derart gibt, daß keine echte direkte Zerlegung $M=M_{0} \oplus M_{1}$ von $M$ mit $M_{0} A=0$ und mit $M_{1} A=M_{1}$ existiert. (Ist $A$ nämlich ein Artinscher halbeinfacher Ring, so gilt für jeden $A$-Modul $M$ eine direkte Zerlegung $M=M_{0} \oplus M_{1}$ mit $M_{0} A=0, \quad M_{1} A=M_{1}$, wegen einer Peirceschen Zerlegung von $M$.) Es sei also $A$ der im Beispiel 4.2 betrachtete Ring $A$ und $M$ die Menge aller Paare $(a, n)$ mit $a \in A, n \in I$, wobei wir 
die Gleichheit und Addition der Paare gewöhnlich definieren. Definiert man auch $(a, n) b=(a b+n b, 0)$, so ist $M$ ein $A$-Modul. Dann folgt aus $M=M_{0} \oplus M_{1}$, aus $M_{0} A=0$ und aus der Halbeinfachheit von $A$ sicher $M_{0}=0$.

SATz 4.4. Ist $A$ ein beliebiger Ring, und ist $x R$ ein direkter Summand von $M$ für jedes in einem Hauptrechtsideale von $A$ liegende Rechtsideal $R$ von $A$ und für jedes Element $x$ von jedem $A$-Modul $M$, so ist $A$ notwendig ein halbeinfacher MHR-Ring.

Beweis. Es sei $A$ ein Ring mit der obigen Eigenschaft und $M$ der spezielle Modul aller Paare $(a, n) \quad(a \in A, n \in I)$ mit $(a, n) b=(a b+n b, 0)$ und mit gewöhnlich definierter Gleichheit und Addition bzw. mit der obigen Operatormultiplikation. Es sei $x=(0,1)$ und $(e, 0)$ die Komponente von $x$ in der Zerlegung $M=x R \oplus K$ für ein Rechtsideal $R$, das in einem Hauptrechtsideale $(a)_{r}(\subseteq A)$ liegt. Dann ist $e(\in A)$ notwendig ein Linkseinselement in $R$, und somit ist $A$ nach unserem Satz 4 in [26] ein halbeinfacher $M H R$ Ring, w. z. b. w.

4.5. Es sei A ein MHR-Ring mit dem Radikal J, und $M$ ein perfekter $A$-Rechstmodul, $K$ ein Untermodul von $M$. Der Faktormodul $M / K$ von $M$ ist dann und nur dann vollständig reduzibel, wenn $M J \subseteq K$ gilt.

BeweIs. Ist $M / K$ vollständig reduzibel, so besteht $(M / K) J=K / K$, also $M J \subseteq K$. Umgekehrt folgt aus $M J \subseteq K$, daß $M / K$ ein (perfekter) $A / J$-Modul ist, der nach 4.3 vollständig reduzibel ist.

4.6. Sind sowohl $M / K_{1}$ als auch $M / K_{2}$ vollständig reduzible, ,ausgezeichnete" Faktormoduln eines perfekten A-Moduls $M$, so gilt $K_{1}=K_{2}$ (Die Definition s. im $§ 1$.)

BEweIs. Unter den Voraussetzungen erhält man nach 4.5 sofort $M J \subseteq K_{1}$ und $M J \subseteq K_{2}$, also $M J \subseteq K_{1} \cap K_{2}$. Dies bedeutet aber nach 4.5, daß $M /\left(K_{1} \cap K_{2}\right)$ ebenfalls vollständig reduzibel ist. $\mathrm{Da}$ auch die $A$-Homomorphismen

$$
m+\left(K_{1} \cap K_{2}\right) \rightarrow m+K_{i} \quad(i=1,2 ; m \in M)
$$

nach der Definition des ausgezeiechneten Faktormoduls Isomorphismen sind, besteht $K_{1}=K_{1} \cap K_{2}=K_{2}$, w. z. b. w.

4.7. Es sei $A$ ein MHR-Ring mit nilpotentem Radikal $J$ und mit der Eigenschaft $a \in a A$ für jedes $a \in A$. Es sei ferner $M=M_{0}$ ein perfekter A-Modul und $M_{k} / M_{k+1}$ ein vollständig reduzibler ausgezeichneter Modul für $k=0,1,2,3, \ldots$ Dann kann das obere Loewysche System $M_{0} \supset M_{1} \supset M_{2} \supset \ldots$ in der kanonischen Form $M \supset M J \supset M J^{2} \supset \ldots$ eindeutig dargestellt werden. Das aus den "Hypersockeln" von $M$ bestehende untere Loewysche System läßt sich aber unter den Voraussetzungen in der kanonischen Gestalt 
$K_{0}(M) \subset K_{1}(M) \subset K_{2}(M) \subset \ldots \quad$ eindeutig darstellen $\quad\left(K_{\gamma}(M)=\{m \mid m \in M\right.$, $\left.\left.m J^{\gamma}=0\right\}\right)^{3}$

BemerkUng 4. 8. Wir wissen bisher noch nicht, wie weit die Voraussetzungen $M A=M, J^{n}=0$ und $a \in a A$ (für jedes $a \in A$ ) dafür abgeschwächt werden können, daß es eine ausführliche Bekanntmachung über eine explizite Darstellung der oberen bzw. unteren Loewyschen Systeme über MHR-Ringe möglich sei. Es ist aber ebenfalls eine offene Frage, ob genau wann die Bedingung $A^{(0)}=A^{(2)}$ für einen halbeinfachen $M H R$-Unterring $A$ des vollen Endomorphismenringes $E$ von einer Abelschen Gruppe $M$ gilt.

\section{$\S 5$. Die verschiedenen Typen der Radikale von $M H R$-Ringen}

Bezeichne $J$ das Jacobsonsche Radikal [13], $B$ das Baer-McCoysche (untere) Nilradikal [2], [19], $N$ das obere Nilradikal, $L$ das Levitzkische Radikal [18], $Z$ das Fuchssche (Zeroid-) Radikal [9] und $G$ das Brown$M c C$ oysche Radikal [3]. In beliebigen Ringen bestehen immer $B \subseteq L \subseteq N \subseteq J \subseteq G$ und $N \subseteq Z$. Es gibt Ringe sowohl mit $Z \subset J$ als auch mit $G \subset Z$. Wir sahen schon bei Satz 1 von [26], daß $J$ in $M H R$-Ringen stets ein Nilideal ist. Vielmehr hat $J$ in $M H R$-Ringen die stärkere Eigenschaft, daR jedes ,,unendliche Produkt" $j_{1} \cdot j_{2} \cdot j_{3} \cdots\left(j_{i} \in J\right)$ gegen Null konvergiert. (Ist aber $A=\{\alpha\}$, $O(a)=0, \quad a^{n}=0 \quad(n \geqq 2)$, so streben alle ,unendlichen Produkte" in $A$ ebenfalls gegen Null, obwohl der Radikalring $A=\{a\}$ kein $M H R$-Ring ist.)

Es gilt nun auch

SATz 5.1. Ist $A$ ein MHR-Ring, so gilt in $A$ immer $B=L=N=J$.

BEwEIs. $A / B$ ist ein Ring ohne nilpotente Ideale $(\neq 0)$ bzw. nilpotente Rechtsideale $(\neq 0)$. Hiernach ist ein in $J / B$ liegendes minimales Rechtsideal weder nilpotent noch idempotent. Dies bedeutet aber $J=B$, also $B=L=$ $=N=J$.

SATZ 5.2. Es gilt in MHR-Ringen A stets $J \subseteq Z$. Hat insbesondere der $M H R$-Ring $A$ ein Rechtseinselement e, so ist $J=Z=G$.

BeweIs. Es sei erstens $A$ ein beliebiger MHR-Ring. Nach [9] ist $Z=Z_{l} \cap Z_{r}$, wobei $Z_{l}$ (bzw. $Z_{r}$ ) die Summe aller $l$-Zeroideale (bzw. $r$-Zeroideale) von $A$ bezeichnet, die mit dem Durchschnitt aller maximalen $l$-Zerofaktorideale (bzw. $r$-Zerofaktorideale) übereinstimmt. Diese maximalen $l$ (bzw. $r$-) Zerofaktorideale sind nach [9] Primideale in $A$. Daher ergibt sich

${ }^{3}$ Vgl. F. SzÁsz, Beziehungen zwischen den Abelschen Gruppen und den assoziativen Ringen mit Minimalbedingung für Hauptrechtsideale, II. Magyar Matematikai Kongresszus (Budapest, 1960), Elöadáskivonatok, Ia, S. 60-62. 
auch $Z \supseteq J$, denn $B$ ist der Durchschnitt aller Primideale, und nach Satz 5. 1 gilt $J=B$.

Es sei nun $A$ ein $M H R$-Ring mit Rechtseinselement $e$. Wir werden beweisen, daß jedes Element $c(\neq 0)$ von jedem echten zweiseitigen Ideal $C(\neq A)$ ein Linksnullteiler ist. Im entgegengesetzten Falle existiert nämlich ein solches minimales Hauptrechtsideal $(d)_{r} \subseteq C$, daß $d$ kein Linksnullteiler von $A$ ist. Hiernach ergibt sich $(d)_{r}=\left(d^{2}\right)_{r}$, also auch $d=d^{2} a(a \in A)$ wegen $e \in A$, weil dann mit $d$ auch $d^{2}$ kein Linksnullteiler in $A$ ist. Da offenbar $e-d a \notin C$ besteht, gilt es auch $e-d a \neq 0$. Dies ist aber unmöglich wegen $d(e-d a)=0$ und wegen der obigen Wahl von $d$. Daher ist jedes echte Ideal $C$ von $A$ ein $l$-Zerofaktorideal, und somit besteht $Z_{l}=J$, denn in $A$ stimmen die primitiven Ideale und die maximalen Ideale untereinander überein (vgl. „Nachträgliche Bemerkungen” unserer Arbeit [26]). Dann gilt aber $Z \subseteq Z_{l}=J \subseteq Z$, also $J=Z\left(=Z_{l} \subseteq Z_{r}\right)$.

Besitzt nun der $M H R$-Ring $A$ ein Rechtseinselement $e$, so ist $A / J$ ein Artinscher halbeinfacher Ring, also halbeinfach auch im Brown-McCoyschen Sinne. Hiernach gilt aber $J=G$, w. z. b. w.

BEISPIELE.

5.3. Ist $A=\{a, b\}$ mit $a+a=b+b=a^{2}=a b=b a=b^{2}+b=0$, so ist $A$ endlich, und zwar $|A|=4$. Ferner gelten $Z=A, B=J=G=\{a\} \neq A$, also $G \subset Z$. $A$ hat kein Rechtseinselement.

5.4. Es sei $A$ der im Beispiel 4.2 betrachtete einfache $M H R$-Ring ohne Rechtseinselement. Dann gelten $B=J=0$ und $G=Z=A$.

5. 5. Ist $A=\{a, b\}$ mit $a+a=b+b=a^{2}+a=b^{2}=b a=a b+b=0$, so gilt im endlichen Ring $A$ ohne Rechtseinselement: $B=J=G=Z=\{b\} \neq A$.

5.6. Ist $A$ der Ring ohne Rechtseinselement aller rationalen Zahlen mit geraden Zählern und mit ungeraden Nennern, so gelten in $A$, der kein $M H R-$ Ring ist, $B=N=Z=0$ und $J=G=A$. Ferner ist (0) in $A$ offenbar ein Primideal, aber weder ein maximales Ideal noch ein primitives Ideal. (Vgl. „Nachträgliche Bemerkungen” unserer Arbeit [26].)

Bemerkungen. Nach den obigen Beispielen kann auch die allgemeine Frage gestellt werden, ob in allen MHR-Ringen das Brown-McCoysche Radikal notwendig ein Teil des Fuchsschen Radikales ist. Man kennt noch ebenfalls kein Kriterium dafür, daß der Faktorring $A / Z$ eines $M H R$-Ringes $A$ nach seinem Fuchsschen Radikal $Z$ halbeinfach im Sinne von Fuchs sei. Da eine transfinite Potenz des Jacobsonschen Radikales $J$ von jedem $M H R$ Ringe $A$ verschwindet, d. h. $J^{\gamma}=0$ mit einer Ordnungszahl $\gamma$ gilt (vgl. [26]), kann es auch folgendes gefragt werden: zu welchen Ordnungszahlen $\gamma$ gibt es wenigstens einen $M H R$-Ring $A$ mit Radikal $J$, für das die Bedingungen 
$J^{\gamma}=0, J^{\beta} \neq 0, \beta<\gamma$ gelten? Dies steht in Beziehung mit dem Problem, ob es zu welchen Ordnungszahlen $\gamma$ wenigstens einen MHR-Ring A mit Rechtseinselement $e$ und mit Radikal $J$ derart gibt, daß der Linksannullator von $J^{\beta}(\beta \leqq \gamma)$ in $A$ genau der $\beta$-te transfinite Rechtssockel von $A$ ist. Es wäre merkwürdig, auch einen $M H R$-Radikalring ohne die Minimalbedingung für Hauptlinksideale zu konstruieren, obwohl wir vermuten, daß ein solcher Radikalring vielleicht überhaupt nicht existieren kann. Bezüglich hierher gehörender eventueller Verallgemeinerungen verweisen wir noch auf eine Arbeit von Herrn H. J. Hoennke (Nilpotenzkriterien, Math. Annolen, 132 (1957), S. $404-411)$.

\section{§ 6. $M M H R$-Ringe und regelmäßige Hauptrechtsideale}

Nach der im $\S 1$ ergebenen Definition ist jeder halbeinfache Artinsche Ring ein MMHR-Ring. Sowohl die ringtheoretische direkte Summe von einfachen Artinschen Ringen (die im allgemeinen kein Artinscher Ring ist), als auch jeder Zeroring $Z\left(p^{\infty}\right)$ ist ein $M H R$-Ring, aber im allgemeinen kein $M M H R$-Ring. Dagegen sind die Artinschen Ringe $A$ ohne $Z\left(p^{\infty}\right)$ in $A^{+}$ immer $M M H R$-Ringe (vgl. FuCHS [7]).

Satz 6. 1. Ist A ein MMHR-Ring mit Radikal J, so ist $A / J$ stets ein Artinscher halbeinfacher Ring. Dann ist die additive Gruppe $A^{+}$von A notwendig die direkte Summe einer torsionsfreien vollständigen Gruppe und endlich vieler ihrer reduzierten p-Komponenten.

BEwEIS. Es genügt zu zeigen, daß jeder halbeinfache MMHR-Ring $B(=A / J)$ ein Artinscher Ring ist. Ist $B=\Sigma \oplus e_{\alpha} B\left(e_{a}^{2}=e_{a}\right)$, so gilt $\left(e_{1}\right)_{r} \subset$ $\subset\left(e_{1}+e_{2}\right)_{r} \subset\left(e_{1}+e_{2}+e_{3}\right)_{r} \subset \ldots$ durch geeignete Wahl der idempotenten Elemente $e_{1}, e_{2}, \ldots$, deren jedes endliche System als ein System paarweise orthogonaler idempotenter Elemente gewählt werden kann. Da $A$ ein $M M H R$ Ring ist, gilt $B=\left(e_{1}+e_{2}+\cdots+e_{s}\right)$, mit einem Index $s$. Hiernach hat $B$ ein Linkseinselement $e_{1}+e_{2}+\cdots+e_{s}$, und deshalb ist $B$ ein halbeinfacher Artinscher Ring.

Ist nun $A$ ein $M H R$-Ring mit $Z\left(p^{\infty}\right) \subseteq A^{+}$, so ist $A$ kein $M M H R$-Ring wegen $Z\left(p^{\infty}\right) \cdot A=0$ nach dem Satz 3.1. Also ist die maximale vollständige Untergruppe eines $M M H R$-Ringes $A$ stets torsionsfrei. Ist ferner $a_{p} \neq 0$ mit $O\left(a_{p}\right)=p$, so gilt $\left(a_{p_{1}}\right)_{r} \subset\left(a_{p_{1}}+a_{p_{2}}\right)_{r} \subset\left(a_{p_{1}}+a_{p_{2}}+a_{p_{3}}\right)_{r} \subset \ldots$ Da $A$ ein MMHR-Ring ist, so hat $A$ tatsächlich nur endlich viele verschiedene $p$-Komponenten. Die Anwendung der Resultate von $\S 3$ beendigt nun den Beweis. 


\section{Bemerkungen und Beispiele.}

1. Die erwähnte Gestalt von $A^{+}$eines $M M H R$-Ringes $A$ ist nur eine notwendige Bedingung bezüglich $A^{+}$über $A^{+}$einen $M M H R$-Ring $A$ zu konstruieren. Sind insbesondere alle $p$-Komponenten $A_{p}^{+}$beschränkt, so ist diese stärkere Bedingung schon sicher hinreichend. Ein genaueres Kriterium ist noch unbekannt.

2. Ist $A$ die direkte Summe aller Zeroringe $Z\left(p^{k}\right) \quad(k=1,2, \ldots$ usw. $)$, so ist $A$ ein $M M H R$-Ring mit unbeschränkter periodischer additiver Gruppe. (Hierbei ist $p$ festgewählt.)

3. Die ringtheoretische direkte Summe $A$ von unendlich vielen Exemplaren der untereinander isomorphen endlichen Primkörper $K_{p}$ ist sicher ein MHR-Ring, aber kein MMHR-Ring, obwohl $p A=0$ gilt. Hier können die Exemplare $K_{p}$ durch die vollen Matrizenringe $\left(K_{p}\right)_{n}(n=1,2,3, \ldots$ usw. $)$ derart ersetzt werden, daß $A$ ebenfalls ein $M H R$-Ring mit $p A=0$ aber kein MMHR-Ring bleibt.

Nun möchten wir die Untersuchungen von DiEUdonné und Hopkins ([5], [12]) über den Sockel $A_{1}$ von $A$ ergänzen und fortentwickeln. Wir benützen dafür den Begriff der regelmäßigen Hauptrechtsideale (s. § 1) und die folgenden Bezeichnungen. Ist $A$ ein beliebiger Ring mit Rechtssockel (kurz nur Sockel) $A_{1}$ und mit den homogenen Komponenten $H_{\mu}$ [13], so gilt $A_{1}=\sum_{\mu \in \Omega} \oplus H_{\mu}$. ( $H_{\mu}$ ist die Summe aller zu einem festen minimalen Rechtsideale $R$ von $A$-isomorphen Rechtsideale von $A$.) Es sei ferner $N_{\mu}$ die Summe aller nilpotenten Rechtsideale von $A$ in $H_{\mu}$, und $M_{\mu}$ ein Rechtsideal von $A$ in $H_{\mu}$ mit $H_{\mu}=M_{\mu} \oplus N_{\mu}$. Bekanntlich ist jedes Rechtsideal sowohl von $M_{\mu}$ als auch von $H_{\mu}$ sicher ein Rechtsideal ebenfalls von $A$ [5]. Es sei nun $N$ die Summe aller $N_{\mu}, N^{*}$ die Summe einiger, endlich vieler, festgewählter $N_{\mu} ; H^{*}$ die Summe der entsprechenden (d. h. zu den in $N^{*}$ liegenden $N_{\mu}$ gehörenden) $H_{\mu}$ und $M^{*}$ die Summe der entsprechenden $M_{\mu}$. Es gilt der folgende

SATz 6.2. I. Ist eA $\left(e^{2}=e\right)$ ein regelmäßiges Hauptrechtsideal von $A$ im Sockel $A_{1}$, so gilt $e A=e A e$, und jedes Rechtsideal von $e A$ ist ein Rechtsideal ebenfalls von A. Ferner ist eA ein Artinscher halbeinfacher Ring, und es gilt auch $e N=0$.

II. Ist nun $N_{0}=N e$ und $n \in N_{0}$, so bestehen $(e+n)^{2}=e+n$ und $e A \oplus N=(e+n) A \oplus N$. Ferner ist $(e+n) A$ mit $e A\left(e^{2}=e, n \in N e\right)$ ebenfalls ein regelmäßiges Hauptrechtsideal. Die Abbildung ex $\rightarrow(e+n) x \quad(x \in A)$ ist ein A-Isomorphismus von $e A$ auf $(e+n) A$ und aus $\left(e+n_{1}\right) A=\left(e+n_{2}\right) A$ folgt notwendig $n_{1}=n_{2}\left(n_{i} \in N e\right)$. Es gelten auch die Beziehungen $A(1-e) \subseteq$ $\subseteq(1-e-n) A$ und $A(1-e-n) \subseteq(1-e) A$. 
III. Besteht umgekehrt $R \oplus N=e A \oplus N$, wobei $R$ ein beliebiges Rechtsideal, eA ein regelmäßiges Hauptrechtsideal von $A$ und $e^{2}=e$ ist, so ist $R$ notwendig ein regelmäßiges Hauptrechtsideal von $A$, und es existiert ein $m \in N e$ mit $R=(e+m) A$.

BeweIs. Es seien $Q_{e}=(1-e) A e A(1-e)$ und $B_{e}=e A(1-e)+$ $+A e A(1-e)$, wobei $e A\left(e^{2}=e\right)$ regelmäßig ist, und in $A_{1}$ liegt. Dann ist $B_{e}$ ein Linksideal von $A$, und es gelten $B_{e}=Q_{e}+e A(1-e)$ und $B_{e}^{k}=Q_{e}^{k}+$ $+e A(1-e) Q_{e}^{k-1}(k=2,3,4, \ldots)$. Da $e A$ regelmäßig ist, existiert ein Exponent $l$ mit $Q_{e}^{l}=0$, also mit $B_{e}^{l+1}=0$. Dann ist aber $D_{e}=e A \cap\left(B_{e}+B_{e} A\right)$ sowohl idempotent wegen $e^{2}=e$ und $D_{e} \subseteq e A \subseteq A_{1}$ als auch nilpotent wegen $B_{e}^{l+1}=0$. Also gelten $D_{e}=0$ und $e A=e A e$ wegen $e A(1-e) \subseteq D_{e}(=0 ;=$ $\left.=e A \cap\left(B_{e}+B_{e} A\right)\right) . A=e A \oplus(1-e) A$ und $e A=e A e\left(e^{2}=e\right)$ bedeuten aber, da $B$ jedes Rechtsideal von $e A$ ebenfalls ein Rechtsideal von $A$ ist. Hiernach ist $e A$ ein halbeinfacher Artinscher Ring.

Aus $e A \cap N=0$ folgt offenbar $e N=0$.

Ist nun $N_{0}=N e$ und $n \in N_{0}$, so gilt $(e+n)^{2}=e+n$ wegen $n e=n$, $e n=0$ und $n^{2}=0$. Da aber $e a=(e+n) a-n a$ und $(e+n) b=e b+n b$ bestehen, ist $e A \oplus N=(e+n) A \oplus N$. Ein elementares Rechnen zeigt nun, da $B$ die Kerne $K_{1}$ bzw. $K_{2}$ der $A$-Homomorphismen $\varphi_{1}: x \rightarrow e x(x \in A)$ bzw. $\varphi_{2}: x \rightarrow(e+n) x \quad(x \in A)$ übereinstimmen, und deshalb ist die Abbildung $\varphi: e x \rightarrow(e+n) x$ ein $A$-Isomorphismus von $e A$ auf $(e+n) A$. (Es gelten nämlich $K_{1} \subseteq K_{2}$ und $K_{2} \subseteq K_{1}$.)

Es seien nun $Q_{e}^{\prime}=0$ und $Q_{e+n}=(1-e-n) A(e+n) A(1-e-n)$. Wir möchten zeigen, daß $Q_{e+n}$ nilpotent, d. h. $(e+n) A$ regelmäßig in $A$ ist. Dies kann wegen $Q_{e+n} \subseteq\left\{Q_{e}, N\right\}$ folgenderweise eingesehen werden. Jedes Element von $\left\{Q_{e}, N\right\}^{2 l}$ ist die Summe gewisser Produkte $P$, deren Faktoren zur mengentheoretischen Vereinigung $Q_{e} \cup N$ gehören. Dann gilt aber sicher $\left\{Q_{e}, N\right\}^{2 l}=0$. Hat nämlich ein Produkt $P$ keinen Faktor aus $N$, so gilt $P \in Q_{e}^{2 l}$, also $P=0$ wegen $Q_{e}^{l}=0$. Besitzt nun ein Produkt $P$ genau einen Faktor $n$ aus $N$, so ist $P=q_{1} q_{2} \ldots q_{s} n q_{1}^{\prime} q_{2}^{\prime} \ldots q_{t}^{\prime}$ mit $q_{i}, q_{j}^{\prime} \in Q_{e}, s \geqq 0, t \geqq 0$. (Das leere Produkt sei nach Definition $1 \in I$.) In diesem Ausdruck ist wegen $(l-1)+1+(l-1)<2 l$ entweder $s \geqq l$ oder $t \geqq l$, und somit gilt auch $P=0$ wegen $Q_{e}^{l}=0$. Enthält drittens ein Produkt $P$ wenigstens zwei Faktoren aus $N$, so ist ebenfalls $P=0$ wegen $N^{2}=0, A N \subseteq N$ und $N A \subseteq N$. Deshalb ist $(e+n) A$ wirklich regelmäßig in $A$, wenn $e A$ das gleiche ist.

Besteht ferner $\left(e+n_{1}\right) A=\left(e+n_{2}\right) A\left(n_{i} \in N_{0}=N e\right)$, wobei $e A \subseteq A_{1}$ regelmäßig ist, so existiert ein $a \in A$ mit $\left(e+n_{1}\right) e=\left(e+n_{2}\right) a$. Folglich ergibt sich $n_{2}-n_{1}=\left(n_{2}-n_{1}\right) e=n_{2} e-\left(e a+n_{2} a-e\right)=\left(e+n_{2}\right)(e-a)$. Hiernach gilt aber $n_{2}-n_{1} \in N \cap\left(e+n_{2}\right) A$, also auch $n_{2}-n_{1}=0$ und $n_{2}=n_{1}$. 
Da die Abbildung $e x \rightarrow(l+u) x$ nach den Vorigen ein $A$-Isomorphismus ist, und $e A=e A e,(e+n) A=(e+n) A(e+n)$ gelten, bestehen auch

$$
\begin{gathered}
A(1-e)=e A(1-e)+(1-e) A(1-e)=(1-e) A(1-e) \subseteq(1-e) A= \\
=(1-e-n) A,
\end{gathered}
$$

und wegen Symmetrie auch $A(1-e-n) \subseteq(1-e) A$. Dies bedeutet aber, daß jeder Linksannullator von $e$ ebenfalls ein Rechtsannullator von $e+n$ ist, bzw. daß jeder Linksannullator von $e+n$ ebenfalls ein Rechtsannullator von $e$ ist.

Gilt zum Schluß $R \oplus N=e A \oplus N$ mit einem beliebigen Rechtsideal $R$ und mit einem regelmäßigen Hauptrechtsideal $e A\left(e^{2}=e\right)$ von $A$ in $A_{1}$, so gilt $e=f+n$ mit $f \in R, n \in N$. Aus $e^{2}=e, N^{2}=0, R N=0$ und $R \cap N=0$ erhält man aber $f^{2}+f n+n f+n^{2}=f+n$, also $f^{2}=f$ und $n f=n$. Da $e=f^{2}+n, f \in R$ und $f A \oplus N \supseteq e A \oplus N=R \oplus N \supseteq f A \oplus N$ bestehen, und der Verband der Untergruppen von $A^{+}$modular ist, ergibt sich $R=f A$ mit $f=e-n$. (Offensichtlich gelten nämlich $f A \subseteq R, \quad f A+N=R+N$ und $f A \cap N=$ $=R \cap N(=0)$, und daher folgt unsere Behauptung.)

SATZ 6.3. Ist insbesondere A entweder ein MMHR-Ring, oder ein Artinscher Ring, so ist $M^{*}$ in A stets ein regelmäßiges Hauptrechtsideal.

Bewers. Da $M^{*}$ die Summe endlich vieler idempotenter minimaler Rechtsideale $e_{1} A, \ldots, e_{n} A\left(e_{i}^{2}=e_{i}\right)$ von $A$ in $H^{*}$ ist, kann es $e_{i+1} \in\left(1-e_{i}\right) \ldots$ $\ldots\left(1-e_{2}\right)\left(1-e_{1}\right) A$ wegen der Anwendung klassischer Methoden vorausgesetzt werden. Hiernach hat aber $M^{*}$ ein Linkseinselement $e$, d. h. $M^{*}=e A\left(e^{2}=e\right)$. Es genügt zu zeigen, daß $Q_{e}=(1-e) A e A(1-e)$ nilpotent ist. Im Falle $d^{*} \in D^{*}=H^{*} \cap(1-e) A$ existieren solche Elemente $a_{1}, a_{2} \in A$ und $n \in N$, daß $d^{*}=e a_{1}+n=(1-e) a_{2}$ ist, denn es gilt $H^{*}=M^{*} \oplus N^{*}$. Nach einer Linksmultiplikation mit $e$ erhält man $e^{2} a_{1}+e n=0$, folglich auch $e a_{1}=0$ wegen $e A \cap N=0$. Dies bedeutet aber, daß $d^{*}=n$, also $D^{*} \subseteq N^{*}$. Hiernach gilt nun $Q_{e}^{2}=0$ wegen $Q_{e} \subseteq D^{*} \subseteq N^{*} \subseteq N$ und wegen $N^{2}=0$, und somit ist $M^{*}$ tatsächlich regelmäßig in $A$.

Bemerkung 6.4. Natürlich kann auch die Frage gestellt werden, ob sich die Voraussetzungen des Satzes 6.2 bzw. des Satzes 6.3 noch wie weit abschwächen lassen. Ferner kennen wir bisher ebenfalls nicht die Existenz eines beliebigen Ringes (bzw. $M H R$-Ringes) $A$ derart, daß $(1-e) A(1-e)$ kein nilpotenter Ring, aber $(1-e) A e A(1-e)$ ein nilpotenter Ring ist. Dies steht in Zusammenhang mit der Möglichkeit, daß sich die „regelmäßigen” Hauptrechtsideale vielleicht auch anders definieren lassen. Es wäre also merkwürdig, diese Beziehungen ausführlicher zu untersuchen. 


\section{§ 7. Über die $M H U$-Ringe}

V. I. Šneľdmyuller [22] betrachtete die Klasse $K$ der Ringe mit Minimalbedingung für alle Unterringe. Jeder Ring aus dieser Klasse $K$ ist ein $M H U$-Ring im Sinne der Definition im § 1. Ein unendlicher Zeroring A mit $p A=0$ gehört nicht zu dieser Klasse $K$, obwohl dieser Ring $A$ ein $M H U$ Ring ist. Ferner haben wir im unseren Satz 3.3 gesehen, daß jeder nilpotente $M H_{1} R$-Ring auch ein $M H U$-Ring ist. Die endlichen Ringe sind natürlich $M H R$-Ringe und auch $M H U$-Ringe. $Z\left(p^{\infty}\right)$ ist ferner ebenfalls sowohl ein $M H R$-Ring als auch ein $M H U$-Ring, obwohl $Z\left(p^{\infty}\right)$ unendlich ist. Dagegen ist der Ring $I$ der ganzen Zahlen weder ein $M H R$-Ring noch ein $M H U$-Ring. Der rationale Zahlkörper $K_{0}$ ist offenbar ein $M H R$-Ring, aber kein $M H U$-Ring. Ist nun $A=\left\{a_{1}, a_{2}, a_{3}, \ldots\right\}$ ein Ring mit $a_{n}+a_{n}=$ $=a_{n}^{n+1}=a_{n} a_{m}+a_{m} a_{n}=0(m \neq n)$, so ist $A$ ein $M H U$-Ring, aber kein MHR-Ring. Gehört nun der Ring $A$ zur Klasse $K^{*}$ aller solchen Ringe, deren jeder endlich erzeugbare echte Unterring ein Hauptrechtsideal in $A$ ist, so ist $A$ genau dann ein $M H U$-Ring, wenn er auch ein $M H R$-Ring ist. Neulich haben wir diese Klasse $K^{*}$ ganz explizit bestimmt (vgl. in speziellem Falle auch Satz 3 von [25]). ${ }^{4}$ Die endlichen Körper, die im allgemeinen nicht zur Klasse $K^{*}$ gehören müssen, zeigen uns die Existenz solcher Ringe, die gleichzeitig $M H R$-Ringe und auch $M H U$-Ringe sind. Jeder unendliche $M H R$-Ring $A$ (MHU-Ring $A$ ) aus der Klasse $K^{*}$ hat eine periodische additive Gruppe $A^{+}$mit $A_{p} \simeq Z\left(p^{\infty}\right)$ bzw. $\simeq Z\left(p^{\infty}\right) \oplus I \mid(p)$ oder $\left|A_{p}\right|<\infty$ für jede Primzahl $p$. Dies ist ein tieferes Korollar von [25].

Die folgenden Tatsachen bezüglich der $M H U$-Ringe können aber viel leichter eingesehen werden. Sowohl jedes homomorphe Bild als auch jeder Unterring eines $M H U$-Ringes ist ebenfalls ein $M H U$-Ring. Ferner hat jeder MHU-Ring $(\neq 0)$ in jedem seiner Unterringe $(\neq 0)$ minimale Unterringe $(\neq 0)$, die Ringe von Primzahlordnung (also Zeroringe oder Primkörper) sind (vgl. Lemma 1 von [24]).

SATZ 7. 1. Die additive Gruppe eines MHU-Ringes ist periodisch. Jeder MHU-Schiefkörper ist ein kommutativer, absolut algebraischer Körper der Charakteristik $p(\neq 0)$. Ist ferner A ein Nilring, der gleichzeitig sowohl ein MHU-Ring als auch ein MHR-Ring ist, so bilden die Elemente von $A$ bezüglich der. Verknüpfung $r_{1} \circ r_{2}=r_{1}+r_{2}-r_{1} r_{2}$ stets eine (nicht notwendig auflösbare) periodische Gruppe G, die das direkte Produkt ihrer Sylowschen p-Untergruppen ist. Die Gruppe $G$ besitzt dann ein transfinites auflösbares invariantes System.

${ }^{4}$ Wir verweisen noch auf die in ${ }^{2}$ zitierte Arbeit. 
Beweis. Ist $P$ das maximale periodische Ideal des $M H U$-Ringes $A$, so ist $(A / P)^{+}$torsionsfrei. Da $A / P$ keinen Unterring einer Ordnung $p$ hat, gilt notwendig $A=P$.

Da der Ring $I$ der ganzen rationalen Zahlen kein $M H U$-Ring ist, hat jeder $M H U$-Schiefkörper $A$ eine Charakteristik $p(\neq 0)$, und besitzt $A$ lauter absolut algebraische Elemente wegen des Abbrechen jeder absteigenden Kette $\{x\} \supset\left\{x^{2}\right\} \supset \cdots \supset\left\{x^{2^{k}}\right\} \supset \ldots$ in $A$. Also ist $A$ nach einem Satz von Jacobson [13] kommutativ, denn es gibt zu jedem $x \in A$ einen Exponenten $n$ mit der Bedingung $x^{n}=x$.

Ist nun $A$ ein $M H U$-Nilring, der gleichzeitig auch ein $M H R$-Ring ist, so bilden die Elemente von $A$ bekanntlich eine Gruppe $G$ bezüglich der Operation $a_{1} \circ a_{2}=a_{1}+a_{2}-a_{1} a_{2}$, denn diese Verknüpfung ist assoziativ. Ferner ist 0 das Einselement dieser Gruppe, und im Falle $a^{n}=0$ ist $b=-\left\{a+a^{2}+\cdots\right.$ $\left.\cdots+a^{n-1}\right)$ das Inverselement von $a$ in $G$. Da im Falle $O^{+}(a)=m(\neq 0, \in I)$ der Unterring $\{a\}$ von $A$ endlich ist, so ist die Ordnung von $a$ in $G$ ebenfalls endlich. Also ist $G$ periodisch. Jede $p$-Komponente $A_{p}$ des Ringes $A$ ist eine Untergruppe in der Gruppe $G$, und zwar kann es gezeigt werden, daß $G$ das direkte Produkt der Sylowschen $p$-Untergruppen $A_{p}$ ist. (Für den Beweis verweisen wir auf [22].) Jedes Ideal von $A$ ist ferner ein Normalteiler in $G$. Da $A$ ein $M H R$-Nilring ist, so existiert eine Ordnungszahl $\gamma$ mit $A^{\gamma}=0, A^{\beta}=0, \beta<\gamma$ (vgl. [26]). Betrachten wir nun das transfinite invariante System $A \supset A^{2} \supset \cdots \supset A^{\omega} \supset \cdots \supset A^{\gamma}=0$, das aus Normalteilern $A^{\beta}$ von $G$ besteht. Dann sind die Faktorgruppen $A^{\beta} / A^{\beta+1}$ bei jedem "Sprung" $(\beta, \beta+1)$ kommutativ wegen $\left(a_{1} \circ A^{\beta+1}\right) \circ\left(a_{2} \circ A^{\beta+1}\right)=a_{1}+a_{2}+A^{\beta+1}=$ $=\left(a_{2} \circ A^{\beta+1}\right) \circ\left(a_{1} \circ A^{\beta+1}\right)$, w. z. b. w.

Bemerkung 7.2. Wir sahen schon, daß die Klassen von $M H R$-Ringen bzw. von $M H U$-Ringen untereinander verschieden sind. Nun stellt sich eine Frage, ob was ein Kriterium dafür ist, daß ein $M H R$-Ring auch ein $M H U$ Ring sei. Es kann auch die umgekehrte Frage betrachtet werden, d. h. genau wann folgt aus der $M H U$-Eigenschaft die $M H R$-Eigenschaft? Diese scheinen uns im allgemeinen schwer.

\section{Anhang}

Zum Schluß möchten wir einige allgemeinere offene Grundfragen bezüglich der $M H R$-Ringe erwähnen, die mit dem Gegenstand unserer Arbeit [26] und der soeben betrachteten Theorie eng zusammenhängen.

Problem 1. Man untersuche die Minimalbedingungen in vollen Matrizenringen $A_{n}(n=2,3,4, \ldots)$ über einem $M H R$-Ring $A$ ! 
Problem 2. Gibt es einen $M H R$-Ring ohne die Minimalbedingung für endlich erzeugte Rechtsideale?

Problem 3. Gibt es einen MHR-Ring mit endlich vielen Hauptrechtsidealen und mit unendlich vielen Hauptlinksidealen?

PROBLEM 4. Was ist ein Kriterium dafür, daß ein $M H R$-Ring auch ein Ring mit Minimalbedingung für Hauptlinksideale sei?

Problem 5. Gibt es einen MHR-Ring ohne die Minimalbedingung für die Potenzen eines festen Hauptrechtsideales?

Problem 6. Genau wann ist ein MHR-Ring auch ein MMHR-Ring?

Problem 7. Welche MHR-Ringe können in einen MHR-Ring mit Einselement eingebettet werden?

PROBlem 8. Man ergebe gewisse Verallgemeinerungen der Theorie von gewöhnlichen primären bzw. vollständig primären Ringe durch [13], durch [26], und durch die Ergebnisse dieser Arbeit!

Problem 9. Man untersuche im Zusammenhang mit Problem 8 die eventuellen verschiedenen direkten Zerlegungen von $M H R$-Ringen $A$, deren Radikal $J$ von Null verschieden ist!

(Eingegangen am 17. Mai 1960.)

\section{Literaturverzeichnis}

[1] E. Artin, C. J. Nesbitt and R. M. Thrall, Rings with minimum condition (Ann Arbor, 1944).

[2] R. Baer, Radical ideals, Amer. Journ. Math., 65 (1943), S. 537-568.

[3] B. Brown and N. H. McCoy, Radicals and subdirect sums, Amer. Journ. Math., 69 (1947), S. 46-58.

[4] M. Deuring, Algebren, Ergebnisse der Math. Wiss. 4 (Berlin, 1947).

[5] J. Dieudonné, Sur le socle d'un anneau et les anneaux simples infinis, Bull. Soc. Math. France, 70 (1942), S. 46-75.

[6] C. C. Falth, Rings with minimum condition on principal ideals, Archiv der Math., $10: 5$ (1959), S. 327-330.

[7] L. Fuchs, Abelian groups (Budapest, 1958).

[8] L. Fuchs, Wann folgt die Maximalbedingung aus der Minimalbedingung?, Archiv der Math., 8 (1957), S. 317-319.

[9] L. Fucrs, On a new type of radical, Acta Sci. Math. Szeged, 16 (1955), S. 43-53.

[10] L. Fuchs and T. Szele, On Artinian rings, Acta Sci. Math. Szeged, 17 (1956), S. 30-40.

[11] A. Gertschiкoff, Über Ringe, die in eine Summe von Körpern zerlegbar sind., Mat. Sbornik, N. S., 7 (1940), S. 591-597 (russisch).

[12] Сн. Норкіns, Rings with minimal condition for left ideals, Annals of Math., 40 (1949), S. $712-730$.

[13y N. Jacobson, Structure of rings, Amer. Math. Soc. Coll. Publ., 37 (1956). 
[14] J. Kaplansky, Topological representation of algebras. II, Trans. Amer. Math. Soc., 68 (1950), S. 62-75.

[15] A. Kertész, Beiträge zur Theorie der Operatormoduln, Acta Math. Acad. Sci. Hung., 8 (1957), S. 235-257.

[16] A. Kertész, Eine Charakterisierung der halbeinfachen Ringe, Acta Math. Acad. Sci. Hung., 9 (1958), S. 343-344.

[17] L. Kovács, A note on regular rings, Publ. Math. Debrecen, 4 (1956), S. $465-468$.

[18] J. Levitzki, On the radical of a general ring, Bull. Amer. Math. Soc., 49 (1943), S. 462-466.

[19] N. H. McCoy, Prime ideals in general rings, Amer. Journ. Math., 71 (1949), S. 823-833.

[20] J. von Neumann, Continuous geometry (Princeton, 1937).

[21] L. RédeI, Algebra. I (Leipzig, 1959).

[22] V. I. Šně̌dmyulere, Unendliche Ringe mit endlichen absteigenden Ketten der Unterringe, Mat. Sbornik, N. S., 27 (1950), S. 219-228 (russisch).

[23] O. Steinfeld, On ideal-quotients and prime ideals, Acta Math. Acad. Sci. Hung., 4 (1953), S. 289-298.

[24] F. SzÁsz, Note on rings in which every proper left-ideal is cyclic, Fund. Math., 44 (1957), S. 330-332.

[25] F. SzÁsz, Die explizite Bestimmung von einigen Klassen der assoziativen Ringe, Bull. Acad. Polon. Sci. Cl. III, 7 (1959), S. 107-110.

[26] F. Szász, Über Ringe mit Minimalbedingung für Hauptrechtsideale. I, Publ. Math. Debrecen, 7 (1960), S. 54-64.

[27] T. Szele, Die Ringe ohne Linksideale, Bul. Stii. Acad. Repub. Popul. Romane, 1 (1949), S. $783-789$.

[28] T. Szele, Nilpotent Artinian rings, Publ. Math. Debrecen, 4 (1955), S. 71-78.

[29] B. L. van der Waerden, Algebra. II (Berlin-Göttingen-Heidelberg, 1955). 